Bull. Korean Math. Soc. 50 (2013), No. 3, pp. 885-899

http://dx.doi.org/10.4134/BKMS.2013.50.3.885

\title{
NOTE ON NULL HELICES IN $\mathbb{E}_{1}^{3}$
}

\author{
Jin Ho Choi And Young Ho Kim
}

\begin{abstract}
In this paper, we study null helices, null slant helices and Cartan slant helices in $\mathbb{E}_{1}^{3}$. Using some associated curves, we characterize the null helices and the Cartan slant helices and construct them. Also, we study a space-like curve with the principal normal vector field which is a degenerate plane curve.
\end{abstract}

\section{Introduction}

In a Lorentzian space, there exist three families of curves which are called space-like, time-like and null (or light-like), depending on their causal characters. It is well-known that the studies of space-like curves and time-like curves have many analogies and similarities because they have the natural geometric invariant parameter by the arc length parameter which normalizes the tangent vector $([22])$. However, the arc length of null curves vanishes and this fact arise the difficulties of study of null curves. As a method of proceeding, some geometers are using the special parameter called the pseudo-arc parameter which normalizes the derivative of the tangent vector $([11,12])$.

In a theory of general relativity, a null curve corresponds to the path of an observer moving at the speed of light while the space-like curves faster and the time-like curves slower than light ([22]). Many mathematical and physical scientists who study the theories of general relativity and classical string emphasized the importance of the null curves in recent years $([6,9,10$, $13,14,15,16,17,18])$.

A helix in $\mathbb{E}^{3}$ (or $\mathbb{E}_{1}^{3}$ ) is defined by a curve whose curvature and torsion are non-zero constants. A certain property of a helix that its tangent lines make a constant angle with a fixed direction leads to the definitions of another types of the helices, for example, the general helices, slant helices and binormal slant helices. Naturally, many mathematicians have been studying another type of

Received March 30, 2012.

2010 Mathematics Subject Classification. Primary 53B30, 53B50, 53C80.

Key words and phrases. Minkowski 3-space, null general helix, null slant helix, Cartan slant helix, associated curve.

Supported by Basic Science Research Program through the National Research Foundation of Korea (NRF) funded by the Ministry of Education, Science and Technology (20100007184). 
helices and they introduced many interesting results different from the ordinary helices (see $[1,2,3,4,5,7,8,20,21]$ ).

In [7], the authors studied also the general helices and the slant helices in $\mathbb{E}^{3}$ by using some special associated curves of a given curve. Subsequently, they studied the Lorentzian version in [8]. They called the special associated curve the principal-directional (-donor) curve and the binormal-directional (-donor) curve. These notions gave us a certain method constructing the general helices and the slant helices (see $[7,8]$ ).

In this paper, we study null helices, Cartan slant helices and null slant helices by using the notions of the associated curves. Also, using the notion of the principal-directional (-donor) curve, we characterize the null helices and the Cartan helices in $\mathbb{E}_{1}^{3}$ and construct them. Finally, we give some properties of a space-like curve with a null principal normal vector field.

\section{Preliminaries}

The Minkowski 3 -space $\mathbb{E}_{1}^{3}$ is the real vector space $\mathbb{R}^{3}$ endowed with the Lorentzian metric given by:

$$
\langle\cdot, \cdot\rangle=-d x_{1}^{2}+d x_{2}^{2}+d x_{3}^{2},
$$

where $\left(x_{1}, x_{2}, x_{3}\right)$ is a rectangular coordinate system of $\mathbb{R}^{3}$. A non-zero vector $v \in \mathbb{E}_{1}^{3}$ is said to be space-like, time-like or null if $\langle v, v\rangle>0,\langle v, v\rangle<0$ or $\langle v, v\rangle=0$, respectively. We consider the zero vector as a space-like vector. Two non-zero vectors $u$ and $v$ in $\mathbb{E}_{1}^{3}$ are said to be orthogonal if $\langle u, v\rangle=0$. A set $\left\{e_{1}, e_{2}, e_{3}\right\}$ of vectors in $\mathbb{E}_{1}^{3}$ is called an orthonormal frame if it satisfies that

$$
\left\langle e_{1}, e_{1}\right\rangle=-1,\left\langle e_{2}, e_{2}\right\rangle=\left\langle e_{3}, e_{3}\right\rangle=1 \text { and }\left\langle e_{i}, e_{j}\right\rangle=0, i \neq j .
$$

For two non-zero vectors $u=\left(u_{1}, u_{2}, u_{3}\right)$ and $v=\left(v_{1}, v_{2}, v_{3}\right)$ in $\mathbb{E}_{1}^{3}$, we define the (Lorentzian) vector product of $u$ and $v$ as the following:

$$
u \times v=\left(u_{3} v_{2}-u_{2} v_{3}, u_{3} v_{1}-u_{1} v_{3}, u_{1} v_{2}-u_{2} v_{1}\right) .
$$

One can check that the vector product is skew-symmetric, i.e., $u \times v=-v \times u$.

A curve $\gamma=\gamma(t)$ in $\mathbb{E}_{1}^{3}$ is said to be space-like, time-like or null if its tangent vector field $\gamma^{\prime}(t)$ is space-like, time-like or null, respectively, for all $t$. A null curve is also called a light-like curve.

Let $\gamma$ be a space-like or time-like curve in $\mathbb{E}_{1}^{3}$ parametrized by arc-length, i.e., $\left|\left\langle\gamma^{\prime}, \gamma^{\prime}\right\rangle\right|=1$, and we suppose that $\left\langle\gamma^{\prime \prime}, \gamma^{\prime \prime}\right\rangle \neq 0$. Then this curve $\gamma$ induces a Frent frame $\left\{T=\gamma^{\prime}, N=\gamma^{\prime \prime} / \sqrt{\left|\left\langle\gamma^{\prime \prime}, \gamma^{\prime \prime}\right\rangle\right|}, B=T \times N\right\}$ satisfying the following Frenet equations:

$$
\left[\begin{array}{l}
T^{\prime} \\
N^{\prime} \\
B^{\prime}
\end{array}\right]=\left[\begin{array}{ccc}
0 & \kappa \eta & 0 \\
-\kappa \epsilon & 0 & -\tau \epsilon \eta \\
0 & -\tau \eta & 0
\end{array}\right]\left[\begin{array}{l}
T \\
N \\
B
\end{array}\right],
$$

where $\epsilon=\langle T, T\rangle, \eta=\langle N, N\rangle, \kappa=\left\langle T^{\prime}, N\right\rangle$ and $\tau=\left\langle N^{\prime}, B\right\rangle$. The vector fields $T, N, B$ and the functions $\kappa$ and $\tau$ are called the tangent, principal normal, 
binormal and curvature and torsion of $\gamma$, respectively. In (2.1), if $\epsilon=1$ or $\epsilon=-1$, then $\gamma$ is space-like or time-like, respectively. A space-like curve $\gamma$ satisfying (2.1) is said to be type 1 or type 2 if $\eta=1$ or $\eta=-1$, respectively.

In case that $\left\langle\gamma^{\prime \prime}, \gamma^{\prime \prime}\right\rangle=0$, if $\gamma^{\prime \prime}=0$, then $\gamma$ is just a space-like straight-line, and if $\gamma^{\prime \prime} \neq 0$, then $\gamma$ is a space-like curve with the null principal normal vector field. In fact, a space-like curve $\gamma$ in $\mathbb{E}_{1}^{3}$ parametrized by arc-length with null principal normal vector field $N$ has a unique null frame $\left\{T=\gamma^{\prime}, N=\gamma^{\prime \prime}, B\right\}$ along $\gamma$ satisfying

$$
\langle N, B\rangle=1,\langle B, B\rangle=\langle T, B\rangle=0
$$

and

$$
\left[\begin{array}{l}
T^{\prime} \\
N^{\prime} \\
B^{\prime}
\end{array}\right]=\left[\begin{array}{ccc}
0 & 1 & 0 \\
0 & k & 0 \\
-1 & 0 & -k
\end{array}\right]\left[\begin{array}{l}
T \\
N \\
B
\end{array}\right],
$$

where $k=\left\langle N^{\prime}, B\right\rangle$. A space-like curve in $\mathbb{E}_{1}^{3}$ satisfying (2.2) is said to be null type.

Let $\gamma=\gamma(t)$ be a non-geodesic null curve in $\mathbb{E}_{1}^{3}$. Then, its acceleration vector $\frac{d^{2} \gamma}{d t^{2}}$ is space-like, i.e., $\left\langle\frac{d^{2} \gamma}{d t^{2}}, \frac{d^{2} \gamma}{d t^{2}}\right\rangle>0$. Hence we can give a reparametrization $s=s(t)$ of $\gamma$ such that $\left\langle\gamma^{\prime \prime}, \gamma^{\prime \prime}\right\rangle=1$, where $\gamma^{\prime \prime}(s)=\frac{d^{2} \gamma}{d s^{2}}$. We call $s$ the pseudo-arc parameter of $\gamma$. With respect to the pseudo-arc parameter $s$, put $A(s)=\gamma^{\prime}(s), C(s)=A^{\prime}(s)$. Then, it is well-known that there is a unique vector field $B(s)$ along $\gamma$ such that $\{A, B, C\}$ forms a null frame along $\gamma$ satisfying (see [10])

$$
A \times B=-C, B \times C=B \text { and } C \times A=A .
$$

The frame $\{A, B, C\}$ satisfies the null Frenet equations:

$$
\left[\begin{array}{l}
A^{\prime} \\
B^{\prime} \\
C^{\prime}
\end{array}\right]=\left[\begin{array}{ccc}
0 & 0 & 1 \\
0 & 0 & k \\
-k & -1 & 0
\end{array}\right]\left[\begin{array}{l}
A \\
B \\
C
\end{array}\right]
$$

where $k=\left\langle B^{\prime}, C\right\rangle$. We call the null frame $\{A, B, C\}$ and the function $k$ the Cartan frame and the light-like curvature of $\gamma$, respectively. The light-like curvature is Lorentzian invariant and the null curves are completely determined only by its light-like curvature up to Lorentz transformations ([11] , [19]). We call the vector fields $B$ and $C$ the binormal vector field and the principal normal vector field along $\gamma$, respectively.

Now, we define some associated curves of a given curve in $\mathbb{E}_{1}^{3}$.

Definition ([7]). Let $\gamma: I \rightarrow \mathbb{E}_{1}^{3}$ be a null curve in $\mathbb{E}_{1}^{3}$ parametrized by the pseudo-arc parameter with Cartan frame $\{A, B, C\}$ and $W$ a unit vector field along $\gamma$. A curve $\bar{\gamma}: I \rightarrow \mathbb{E}_{1}^{3}$ is called the $W$-directional curve of the curve $\gamma$ if the tangent $\bar{T}$ of the curve $\bar{\gamma}$ is equal to $W$, i.e., $\bar{T}=W$. A curve $\gamma$ whose $W$-directional curve $\bar{\gamma}$ is called the $W$-donor curve of $\bar{\gamma}$. 
Remark 2.1. For a given curve in $\mathbb{E}_{1}^{3}$, its $W$-directional and $W$-donor curves are unique up to translations (For the details, see [7]).

Remark 2.2. If $W=A$, then the $A$-directional curve $\bar{\gamma}$ of the curve $\gamma$ is trivially $\gamma$.

Remark 2.3. If $W=B$, then $\bar{A}=B$. In this case the $B$-directional curve $\bar{\gamma}$ of the curve $\gamma$ is called the binormal-directional curve of $\gamma$ and the curve $\gamma$ is called the binormal-donor curve of $\bar{\gamma}$. When a curve $\overline{\bar{\gamma}}$ is the binormaldirectional curve of $\bar{\gamma}$, we call $\overline{\bar{\gamma}}$ the second principal-directional curve of $\gamma$ and $\gamma$ the second binormal-donor curve of $\overline{\bar{\gamma}}$.

Remark 2.4. If $W=C$, then $\bar{A}=C$. In this case the $C$-directional curve $\bar{\gamma}$ of the curve $\gamma$ is called the principal-directional curve of $\gamma$ and the curve $\gamma$ is called the principal-donor curve of $\bar{\gamma}$.

\section{Principal-directional curve of null curves and Cartan slant helix}

A null helix in $\mathbb{E}_{1}^{3}$ is a null curve with constant light-like curvature. Equivalently, a null helix $\gamma$ is a null curve that there exists a vector $V \in \mathbb{E}_{1}^{3}$ such that $\left\langle\gamma^{\prime}, V\right\rangle$ is constant ([12], [19]).

Let $\{A, B, C\}$ be the Cartan frame along a given null curve $\gamma$. By definition, a Cartan slant helix (resp. null slant helix) in $\mathbb{E}_{1}^{3}$ is the one with a vector $V \in \mathbb{E}_{1}^{3}$ such that $\langle C, V\rangle$ (resp. $\langle B, V\rangle)$ is constant.

In fact, the notions of the principal-directional curve and the principal-donor curve are very useful to study the relationship between null helices and the Cartan slant helices (or null slant helices) in $\mathbb{E}_{1}^{3}$. The following remarks explain why.

Remark 3.1. Let $\gamma=\gamma(s)$ be a curve in $\mathbb{E}_{1}^{3}$ and $W=W(s)$ a unit vector field along $\gamma$. If $W$ has a constant angle with a constant vector $V$ of $\mathbb{E}_{1}^{3}$ along $\gamma$, then the tangent vector of the $W$-directional curve $\bar{\gamma}$ of $\gamma$ has also a constant angle with $V$ along $\bar{\gamma}$. Conversely, for a curve $\bar{\gamma}$ in $\mathbb{E}_{1}^{3}$, if its tangent has a constant angle with a constant vector field $V$ of $\mathbb{E}_{1}^{3}$, then its $W$-donor curve $\gamma$ is a curve in $\mathbb{E}_{1}^{3}$ satisfying the condition that $W$ has constant angle with $V$ along $\bar{\gamma}$.

Remark 3.2. In Remark 3.1, if we take the principal normal vector field $C$ (resp. the binormal vector field $B$ ) instead of the vector field $W$ along $\gamma$, then $\gamma$ is a Cartan slant helix (resp. null slant helix) in $\mathbb{E}_{1}^{3}$. In other words, a Cartan slant helix (resp. null slant helix) in $\mathbb{E}_{1}^{3}$ is a principal (resp. binormal)donor curve of a null helix in $\mathbb{E}_{1}^{3}$ and a null helix in $\mathbb{E}_{1}^{3}$ is a principal (resp. binormal)-directional curve of a Cartan slant helix (resp. null slant helix) in $\mathbb{E}_{1}^{3}$.

Now, we give the relationship between a null curve $\gamma$ parametrized by the pseudo-arc parameter $s$ and its principal-directional curve $\bar{\gamma}$ in $\mathbb{E}_{1}^{3}$. 
Theorem 3.3. Let $\gamma$ be a null curve in $\mathbb{E}_{1}^{3}$ parametrized by the pseudo-arc parameter $s$ with the light-like curvature $k \neq 0$ and $\bar{\gamma}$ the principal-directional curve of $\gamma$. If $k>0$ or $k<0$, then $\bar{\gamma}$ is a space-like curve of type 1 or type 2 , respectively. Moreover, for the curvature $\bar{\kappa}$ and the torsion $\bar{\tau}$ of $\bar{\gamma}$, it has the following relations:

$$
\bar{\kappa}=\sqrt{2|k|}, \quad \bar{\tau}=-\epsilon \frac{k^{\prime}}{2 k} \text { and } \frac{\bar{\tau}}{\bar{\kappa}}=\epsilon\left(\frac{1}{\sqrt{2|k|}}\right)^{\prime},
$$

where $\epsilon$ is 1 or -1 if $k>0$ or $k<0$, respectively.

Proof. Since $\bar{\gamma}$ is the principal-directional curve of $\gamma, \bar{\gamma}^{\prime}=\bar{T}=C$. We note that the parameter $s$ is the arc-length parameter along $\bar{\gamma}$, i.e., $\bar{s}=s$. Differentiation of $\bar{T}$ along the curve $\bar{\gamma}$ leads to

$$
\frac{d \bar{T}}{d \bar{s}}=\frac{d C}{d \bar{s}}=\frac{d C}{d s}=-k A-B
$$

Since $\left\langle\frac{d \bar{T}}{d \bar{s}}, \frac{d \bar{T}}{d \bar{s}}\right\rangle=2 k$, the type of the space-like curve $\bar{\gamma}$ depends on the sign of the light-like curvature of $\gamma$. The curvature $\bar{\kappa}$ of $\bar{\gamma}$ and the principal normal vector field $\bar{N}$ along $\bar{\gamma}$ are easily derived as

$$
\bar{\kappa}=\sqrt{2|k|} \text { and } \bar{N}=\frac{1}{\sqrt{2|k|}}\{-k A-B\},
$$

respectively. Since $\bar{T}=C$, from equation (3.1), we can find the binormal vector field $\bar{B}$ along $\bar{\gamma}$ :

$$
\begin{aligned}
\bar{B} & =-\epsilon(\bar{T} \times \bar{N})=\frac{\epsilon}{\sqrt{2|k|}} C \times(k A+B) \\
& =\frac{\epsilon}{\sqrt{2|k|}}\{k A-B\},
\end{aligned}
$$

where $\epsilon$ is the sign of $k$. By the direct calculation, we have

$$
\frac{d \bar{B}}{d \bar{s}}=\epsilon\left\{\frac{k^{\prime}}{2 k \sqrt{2|k|}}(k A+B)\right\} .
$$

Thus, we get the torsion $\bar{\tau}$ of $\bar{\gamma}$ which is given by

$$
\bar{\tau}=-\epsilon \frac{k^{\prime}}{2 k} \text {. }
$$

Therefore, we have

$$
\frac{\bar{\tau}}{\bar{\kappa}}=-\frac{\epsilon k^{\prime}}{2 k \sqrt{2|k|}}=\epsilon\left(\frac{1}{\sqrt{2|k|}}\right)^{\prime} .
$$

Thus, our proof is completed.

For a given null curve in $\mathbb{E}_{1}^{3}$ parametrized by the pseudo-arc parameter $s$, we showed that its principal-directional curve is always a space-like Frenet curve. But, the converse is not true. In other words, a principal-donor curve of a 
space-like Frenet curve in $\mathbb{E}_{1}^{3}$ need not be a null curve parametrized by the pseudo-arc parameter. In fact, from Theorem 3.3, we get the following:

Corollary 3.4. Let $\bar{\gamma}$ be a space-like curve in $\mathbb{E}_{1}^{3}$ with the non-zero curvature $\bar{\kappa}$ and the torsion $\bar{\tau}$ and $\{\bar{T}, \bar{N}, \bar{B}\}$ the Frent frame of $\bar{\gamma}$. Then, a principaldonor curve $\gamma$ of $\bar{\gamma}$ is a null curve parametrized by pseudo-arc parameter if and only if $\bar{\kappa}$ and $\bar{\tau}$ satisfy $\frac{\bar{\tau}}{\bar{\kappa}}=\epsilon\left(\frac{1}{\bar{\kappa}}\right)^{\prime}$, where $\epsilon=\langle\bar{N}, \bar{N}\rangle$. Moreover, the light-like curvature of $\gamma$ is given by $k(s)=\epsilon \frac{\bar{\kappa}^{2}}{2}$.

Proof. If a principal-donor curve $\gamma$ is a null curve parametrized by pseudo-arc parameter, it is clear from Theorem 3.3. Conversely, assume that the curve $\bar{\gamma}$ satisfies $\frac{\bar{\tau}}{\bar{\kappa}}=\epsilon\left(\frac{1}{\bar{\kappa}}\right)^{\prime}$ for $\epsilon=\langle\bar{N}, \bar{N}\rangle$. From equations (3.1) and (3.2), a principaldonor curve $\gamma$ of a space-like curve $\bar{\gamma}$ in $\mathbb{E}_{1}^{3}$ is constructed by

$$
\gamma^{\prime}=A=\frac{1}{\bar{\kappa}}\{-\epsilon \bar{N}+\bar{B}\},
$$

where $\epsilon=\langle\bar{N}, \bar{N}\rangle=-\langle\bar{B}, \bar{B}\rangle$. Trivially, the curve $\gamma$ is null. Differentiating (3.3), we have

$$
\begin{aligned}
C & =\gamma^{\prime \prime}=\left(\frac{1}{\bar{\kappa}}\right)^{\prime}\{-\epsilon \bar{N}+\bar{B}\}+\frac{1}{\bar{\kappa}}\{\bar{\kappa} \bar{T}-\epsilon \bar{\tau} \bar{B}+\bar{\tau} \bar{N}\} \\
& =\bar{T}+\left(\frac{\bar{\tau}}{\bar{\kappa}}-\epsilon\left(\frac{1}{\bar{\kappa}}\right)^{\prime}\right) \bar{N}+\left(\left(\frac{1}{\bar{\kappa}}\right)^{\prime}-\epsilon \frac{\bar{\tau}}{\bar{\kappa}}\right) \bar{B}=\bar{T} .
\end{aligned}
$$

From equation (3.3), the principal vector field $B$ along $\gamma$ could be given by ( $c f$. [10], page 10)

$$
\begin{aligned}
B & =\frac{1}{g(A, \bar{B})}\left\{\bar{B}-\frac{g(\bar{B}, \bar{B})}{2 g(A, \bar{B})} A\right\} \\
& =-\epsilon \bar{\kappa}\left\{\bar{B}-\frac{1}{2}(-\epsilon \bar{N}+\bar{B})\right\} \\
& =-\epsilon \frac{\bar{\kappa}}{2}\{\bar{B}+\epsilon \bar{N}\},
\end{aligned}
$$

from which, together with $\frac{\bar{\tau}}{\bar{\kappa}}=\epsilon\left(\frac{1}{\bar{\kappa}}\right)^{\prime}$, we get

$$
B^{\prime}=\epsilon \frac{\bar{\kappa}^{2}}{2} \bar{T}
$$

Therefore, we have $k(s)=\epsilon \frac{\bar{\kappa}^{2}}{2}$.

This leads to a characterization of null helices in $\mathbb{E}_{1}^{3}$ as follows:

Corollary 3.5. A null curve in $\mathbb{E}_{1}^{3}$ parametrized by the pseudo-arc parameter $s$ with the light-like curvature $k \neq 0$ is a null helix if and only if its principal-directional curve $\bar{\gamma}$ is a plane curve of the constant curvature. Moreover, depending on $\kappa>0$ or $\kappa<0, \bar{\gamma}$ is a space-like circle in $\mathbb{E}^{2}$ or a space-like hyperbola in $\mathbb{E}_{1}^{2}$, respectively. 
Together with Corollary 3.5, equation (3.3) gives an expression of the general helices in $\mathbb{E}_{1}^{3}$.

Corollary 3.6 ([12]). Let $\gamma$ be a null general helix in $\mathbb{E}_{1}^{3}$ with the light-like curvature $k \neq 0$.

(a) If $k$ is positive, then $\gamma$ can be expressed by

$$
\gamma(s)=\frac{1}{2 k}(-\sqrt{2 k} s, \sin [\sqrt{2 k} s],-\cos [\sqrt{2 k} s]) .
$$

(b) If $k$ is negative, then $\gamma$ can be expressed by

$$
\gamma(s)=\frac{1}{2 k}(\sinh [\sqrt{-2 k} s], \cosh [\sqrt{-2 k} s], \sqrt{-2 k} s) .
$$

Proof. Let $\bar{\gamma}$ be a space-like circle in $\mathbb{E}^{2}$ with the constant curvature $\bar{\kappa}$ as a principal-directional curve of the given curve $\gamma$. Then, $\bar{\gamma}$ can be expressed by

$$
\bar{\gamma}(s)=\frac{1}{\bar{\kappa}}(0, \cos [\bar{\kappa} s], \sin [\bar{\kappa} s]),
$$

from which, we have the Frenet frame $\{\bar{T}, \bar{N}, \bar{B}\}$ along $\bar{\gamma}$ as follows:

$$
\left\{\begin{array}{l}
\bar{T}(s)=(0,-\sin [\bar{\kappa} s], \cos [\bar{\kappa} s]), \\
\bar{N}(s)=(0,-\cos [\bar{\kappa} s],-\sin [\bar{\kappa} s]), \\
\bar{B}(s)=(-1,0,0) .
\end{array}\right.
$$

From equation (3.3), we have

$$
\begin{aligned}
\gamma^{\prime}(s) & =\frac{1}{\bar{\kappa}}\{-\bar{N}+\bar{B}\} \\
& \left.=\frac{1}{\bar{\kappa}}(-1, \cos [\bar{\kappa} s], \sin [\bar{\kappa} s]]\right) \\
& =\frac{1}{\sqrt{2 k}}(-1, \cos [\sqrt{2 k} s], \sin [\sqrt{2 k} s]),
\end{aligned}
$$

where the last equality come from (3.1). Since $k$ is a constant, we get the first expression (3.4). The proof of (b) is similar to (a).

Remark 3.7. In Corollary 3.4, if the curve $\bar{\gamma}$ is a general helix with $\bar{\tau}=m \bar{\kappa}$, then the curvature function $\bar{\kappa}$ is equal to $\frac{1}{m s+b}$. Also, the light-like curvature $k$ of $\gamma$ is given by $k(s)=\frac{\epsilon}{2(m s+b)^{2}}$.

From Remark 3.7, we can give a characterization of Cartan slant helix in $\mathbb{E}_{1}^{3}$.

Theorem 3.8. Let $\gamma$ is a null curve in $\mathbb{E}_{1}^{3}$ parametrized by the pseudo-arc parameter $s$. Then, the followings are equivalent:

(a) $\gamma$ is a Cartan slant helix in $\mathbb{E}_{1}^{3}$.

(b) $\gamma$ has the light-like curvature $\pm \frac{1}{(c s+b)^{2}}$, where $c \neq 0$ and $b$ are constants.

(c) The principal-directional curve $\bar{\gamma}$ of $\gamma$ is a space-like general helix $\bar{\gamma}$ with $\bar{\kappa}(s)=\frac{1}{m s+a}$ and $\bar{\tau}(s)=\frac{m}{m s+a}$, where $m=\frac{c}{\sqrt{2}}$ and $a=\frac{b}{\sqrt{2}}$. 
(d) $\gamma$ is the principal-donor curve of a space-like general helix $\bar{\gamma}$ with $\bar{\kappa}(s)=$ $\frac{1}{m s+a}$ and $\bar{\tau}(s)=\frac{m}{m s+a}$, where $m=\frac{c}{\sqrt{2}}$ and $a=\frac{b}{\sqrt{2}}$.

Finally, motivated by Theorem 3.8(d), we can construct a Cartan slant helix in $\mathbb{E}_{1}^{3}$ from a space-like general helix. To do this, we need the following two lemmas:

Lemma $3.9([8])$. Let $\gamma$ be a space-like general helix $\gamma$ of type 1 in $\mathbb{E}_{1}^{3}$ satisfying $\tau=m \kappa$.

(a) If $\left|\frac{\tau}{\kappa}\right|=|m|<1$, then $\gamma$ can be locally expressed by

$$
\gamma(s)=\frac{1}{\sqrt{1-m^{2}}} \int\left(m, \sin \left[\sqrt{1-m^{2}} \int \kappa(s) d s\right],-\cos \left[\sqrt{1-m^{2}} \int \kappa(s) d s\right]\right) d s .
$$

(b) If $\left|\frac{\tau}{\kappa}\right|=|m|>1$, then $\gamma$ can be locally expressed by

$$
\gamma(s)=\frac{-1}{\sqrt{m^{2}-1}} \int\left(\cosh \left[\sqrt{m^{2}-1} \int \kappa(s) d s\right], \sinh \left[\sqrt{m^{2}-1} \int \kappa(s) d s\right], m\right) d s .
$$

Lemma 3.10 ([8]). A space-like generalized helix $\gamma$ of type 2 in $\mathbb{E}_{1}^{3}$ satisfying $\tau=m \kappa$ is locally expressed by

$$
\gamma(s)=\frac{1}{\sqrt{1+m^{2}}} \int\left(\sinh \left[\sqrt{1+m^{2}} \int \kappa(s) d s\right], \cosh \left[\sqrt{1+m^{2}} \int \kappa(s) d s\right], m\right) d s .
$$

From Lemmas 3.9 and 3.10, we know that there are three different types of Cartan slant helices in $\mathbb{E}_{1}^{3}$.

Now, we can construct the Cartan slant helices from (3.8), (3.9) and (3.10), respectively.

Theorem 3.11. Let $\gamma$ be a Cartan slant helix $\gamma$ with the light-like curvature $k(s)=\frac{\epsilon}{(c s+b)^{2}}(\epsilon= \pm 1)$.

(a) If $\epsilon=1$ and $|c|<\sqrt{2}$, then $\gamma(s)=\left(x_{1}(s), y_{1}(s), z_{1}(s)\right)$ can be expressed by

$$
\begin{aligned}
x_{1}(s)=- & \frac{s(c s+2 b)}{2 \sqrt{2-c^{2}}} \\
y_{1}(s)=- & \frac{(c s+b)^{2}}{\sqrt{2} \sqrt{2-c^{2}}\left(2+3 c^{2}\right)}\left(c \sqrt{2-c^{2}} \cos \left[\frac{\sqrt{2-c^{2}}}{c} \ln (c s+b)\right]\right. \\
+ & \left.\left(2+c^{2}\right) \sin \left[\frac{\sqrt{2-c^{2}}}{c} \ln (c s+b)\right]\right), \\
z_{1}(s)=- & \frac{(c s+b)^{2}}{\sqrt{2} \sqrt{2-c^{2}}\left(2+3 c^{2}\right)}\left(c \sqrt{2-c^{2}} \sin \left[\frac{\sqrt{2-c^{2}}}{c} \ln (c s+b)\right]\right. \\
& \left.-\left(2+c^{2}\right) \cos \left[\frac{\sqrt{2-c^{2}}}{c} \ln (c s+b)\right]\right) .
\end{aligned}
$$


(b) If $\epsilon=1$ and $|c|>\sqrt{2}$, then $\gamma(s)=\left(x_{2}(s), y_{2}(s), z_{2}(s)\right)$ can be expressed by

$$
\begin{aligned}
x_{2}(s)= & \frac{(c s+b)^{2}}{\sqrt{2} \sqrt{c^{2}-2}\left(2+3 c^{2}\right)}\left(\left(c^{2}+2\right) \cosh \left[\frac{\sqrt{c^{2}-2}}{c} \ln (c s+b)\right]\right. \\
& \left.+c \sqrt{c^{2}-2} \sinh \left[\frac{\sqrt{c^{2}-2}}{c} \ln (c s+b)\right]\right), \\
y_{2}(s)= & \frac{(c s+b)^{2}}{\sqrt{2} \sqrt{c^{2}-2}\left(2+3 c^{2}\right)}\left(\left(c^{2}+2\right) \sinh \left[\frac{\sqrt{c^{2}-2}}{c} \ln (c s+b)\right]\right. \\
& \left.+c \sqrt{c^{2}-2} \cosh \left[\frac{\sqrt{c^{2}-2}}{c} \ln (c s+b)\right]\right), \\
z_{2}(s)= & \frac{s(c s+2 b)}{2 \sqrt{c^{2}-2}} .
\end{aligned}
$$

(c) If $\epsilon=-1$, then $\gamma(s)=\left(x_{3}(s), y_{3}(s), z_{3}(s)\right)$ can be expressed by

$$
\begin{aligned}
x_{3}(s)= & \frac{(c s+b)^{2}}{\sqrt{2} \sqrt{c^{2}+2}\left(3 c^{2}-2\right)}\left(\left(c^{2}-2\right) \sinh \left[\frac{\sqrt{c^{2}+2}}{c} \ln (c s+b)\right]\right. \\
& \left.+c \sqrt{c^{2}+2} \cosh \left[\frac{\sqrt{c^{2}+2}}{c} \ln (c s+b)\right]\right), \\
y_{3}(s)= & \frac{(c s+b)^{2}}{\sqrt{2} \sqrt{c^{2}+2}\left(3 c^{2}-2\right)}\left(\left(c^{2}-2\right) \cosh \left[\frac{\sqrt{c^{2}+2}}{c} \ln (c s+b)\right]\right. \\
& \left.+c \sqrt{c^{2}+2} \sinh \left[\frac{\sqrt{c^{2}+2}}{c} \ln (c s+b)\right]\right), \\
z_{3}(s)= & -\frac{s(c s+2 b)}{2 \sqrt{c^{2}+2}} .
\end{aligned}
$$

Proof. (a) Since $k>0$, from Theorem 3.3, its principal-directional curve $\bar{\gamma}$ is a space-like curve of type 1 . Also, from Theorem 3.8, we know that $\bar{\gamma}$ has the curvature $\bar{\kappa}=\frac{\sqrt{2}}{c s+b}$ and the torsion $\bar{\tau}=\frac{c}{c s+b}$. Since $|c|<\sqrt{2}$, we have $\left|\frac{\bar{\tau}}{\bar{\kappa}}\right|=\left|\frac{c}{\sqrt{2}}\right|<1$ and $\bar{\gamma}$ is expressed by (3.8) from Lemma 3.9(a). By the direct calculation, we get the Frenet frame $\{\bar{T}, \bar{N}, \bar{B}\}$ along $\bar{\gamma}$ given by

$$
\left\{\begin{array}{l}
\bar{T}(s)=\frac{\sqrt{2}}{\sqrt{2-c^{2}}}\left(\frac{c}{\sqrt{2}}, \sin \left[\frac{\sqrt{2-c^{2}}}{c} \ln (c s+b)\right],-\cos \left[\frac{\sqrt{2-c^{2}}}{c} \ln (c s+b)\right]\right), \\
\bar{N}(s)=\left(0, \cos \left[\frac{\sqrt{2-c^{2}}}{c} \ln (c s+b)\right], \sin \left[\frac{\sqrt{2-c^{2}}}{c} \ln (c s+b)\right]\right), \\
\bar{B}(s)=\frac{-1}{\sqrt{2-c^{2}}}\left(\sqrt{2}, c \sin \left[\frac{\sqrt{2-c^{2}}}{c} \ln (c s+b)\right],-c \cos \left[\frac{\sqrt{2-c^{2}}}{c} \ln (c s+b)\right]\right) .
\end{array}\right.
$$


With this, equation (3.3) implies

$$
\begin{aligned}
& \gamma^{\prime}(s)=\frac{1}{\bar{\kappa}}\{-\bar{N}+\bar{B}\} \\
& =-\frac{c s+b}{\sqrt{2}}\left(\frac{\sqrt{2}}{\sqrt{2-c^{2}}},\right. \\
& \cos \left[\frac{\sqrt{2-c^{2}}}{c} \ln (c s+b)\right]+\frac{c}{\sqrt{2-c^{2}}} \sin \left[\frac{\sqrt{2-c^{2}}}{c} \ln (c s+b)\right], \\
& \left.\sin \left[\frac{\sqrt{2-c^{2}}}{c} \ln (c s+b)\right]-\frac{c}{\sqrt{2-c^{2}}} \cos \left[\frac{\sqrt{2-c^{2}}}{c} \ln (c s+b)\right]\right) \text {. }
\end{aligned}
$$

One can check that

$$
\begin{aligned}
& \int(c s+b) \sin \left[\frac{\sqrt{2-c^{2}}}{c} \ln (c s+b)\right] \\
= & \frac{(c s+b)^{2}}{3 c^{2}+2}\left(2 c \sin \left[\frac{\sqrt{2-c^{2}}}{c} \ln (c s+b)\right]-\sqrt{2-c^{2}} \cos \left[\frac{\sqrt{2-c^{2}}}{c} \ln (c s+b)\right]\right)
\end{aligned}
$$

and

$$
\begin{aligned}
& \int(c s+b) \cos \left[\frac{\sqrt{2-c^{2}}}{c} \ln (c s+b)\right] \\
= & \frac{(c s+b)^{2}}{3 c^{2}+2}\left(2 c \cos \left[\frac{\sqrt{2-c^{2}}}{c} \ln (c s+b)\right]+\sqrt{2-c^{2}} \sin \left[\frac{\sqrt{2-c^{2}}}{c} \ln (c s+b)\right]\right) .
\end{aligned}
$$

Thus, (a) is obtained.

Similarly, (b) and (c) are obtained.

\section{Binormal-directional curve of null curves and null slant helices}

In $\S 2$, we defined a null slant helix in $\mathbb{E}_{1}^{3}$ as another type of a helix. However, it is well-known that the null helix and the null slant helix make no difference $([12])$. At the end of this section, we prove this fact as a corollary of our theorem.

The following theorem gives a relationship between a null curve and its null binormal-directional curve $\bar{\gamma}$ in $\mathbb{E}_{1}^{3}$. Note that the parameter $s$ of $\gamma$ need not be the pseudo-arc parameter of $\bar{\gamma}$.

Theorem 4.1. Let $\gamma$ be a null curve in $\mathbb{E}_{1}^{3}$ parametrized by the pseudo-arc parameter $s$ with the non-zero light-like curvature $k$ and $\bar{\gamma}$ its null binormaldirectional curve with the light-like curvature $\bar{k}$. Then, it satisfies that

$$
\bar{k}=\frac{1}{k} .
$$


Proof. Let $\{A, B, C\}$ be a Cartan frame of a null curve $\gamma$ parametrized by pseudo-arc parameter $s$ and $\{\bar{A}, \bar{B}, \bar{C}\}$ a Cartan frame of the null binormaldirectional curve $\bar{\gamma}$ of pseudo-arc parameter $\bar{s}$ in $\mathbb{E}_{1}^{3}$. This means that two curves $\gamma$ and $\bar{\gamma}$ have the null Frenet equations:

$$
\frac{d A}{d s}(s)=C(s), \frac{d B}{d s}(s)=k(s) C(s), \frac{d C}{d s}(s)=-k(s) A(s)-B(s)
$$

and

$$
\frac{d \bar{A}}{d \bar{s}}(\bar{s})=\bar{C}(\bar{s}), \frac{d \bar{B}}{d \bar{s}}(\bar{s})=\bar{k}(\bar{s}) \bar{C}(\bar{s}), \frac{d \bar{C}}{d \bar{s}}(\bar{s})=-\bar{k}(\bar{s}) \bar{A}(\bar{s})-\bar{B}(\bar{s})
$$

From our assumption, we have $\bar{A}(\bar{s}(s))=B(s)$. Then, by (4.1) and (4.2), we have

$$
\bar{C}(\bar{s})=\frac{d \bar{A}}{d \bar{s}}=\frac{d B}{d \bar{s}}=\frac{d s}{d \bar{s}} \frac{d B}{d s}=\frac{d s}{d \bar{s}} \cdot(k(s) C(s)) .
$$

Since $\bar{C}$ and $C$ are unit space-like vectors in $\mathbb{E}_{1}^{3}$, we get that $k(s)= \pm d \bar{s} / d s$ and $\bar{C}(\bar{s}(s))= \pm C(s)$. The derivative of $\bar{C}$ with respect to $\bar{s}$ leads to

$$
-\bar{k} \bar{A}-\bar{B}=\frac{d \bar{C}}{d \bar{s}}=\epsilon \frac{d C}{d \bar{s}}=\epsilon \frac{d s}{d \bar{s}} \frac{d C}{d s}=\frac{1}{k}\{-k A-B\}=-A-\frac{1}{k} B,
$$

where $\epsilon=-1$ or 1 . Since $\bar{A}=B$, we get from (4.3) that

$$
\left(\frac{1}{k}-\bar{k}\right) B+A=\bar{B}
$$

Thus, we have $\bar{k}=1 / k$.

Corollary 4.2 ([20]). A null curve $\gamma$ in $\mathbb{E}_{1}^{3}$ is a null slant helix if and only if $\gamma$ is a null helix.

\section{Space-like curves of null type}

For a non-null curve $\gamma$ in $\mathbb{E}_{1}^{3}, \gamma$ is a general helix, slant helix or binormal slant helix if there exists a non-zero vector $V \in \mathbb{E}_{1}^{3}$ such that $\langle T, V\rangle,\langle N, V\rangle$ or $\langle B, V\rangle$ is constant, respectively, where $\{T, N, B\}$ is the Frenet frame of $\gamma$. In case of non-null curve with the non-null principal normal vector field, it is easily seen that plane curves are general helices, slant helices and binormal slant helices. In fact, the binormal vector field $B$ along $\gamma$ is constant and we see $\langle T, B\rangle=\langle N, B\rangle=0$ and $\langle B, B\rangle=1$.

In this section, we will show that a space-like curve of null type is a degenerate plane curve, but the curve need not be a binormal slant helix.

Remark 5.1. Any curve in a degenerate plane of $\mathbb{E}_{1}^{3}$ is either a space-like curve of null type or a null straight-line. 
Let $\gamma$ be a space-like curve of null type in $\mathbb{E}_{1}^{3}$ and $\{T, N, B\}$ the Frenet frame satisfying (2.2) along $\gamma$. Since $N^{\prime}(s)=k(s) N(s)$, the null principal normal vector field $N$ along $\gamma$ is given by

$$
N(s)=\exp \left(\int k(s) d s\right) \mathbf{b},
$$

where $\mathbf{b}$ is a null vector in $\mathbb{E}_{1}^{3}$. Since $\gamma^{\prime \prime}(s)=T^{\prime}(s)=N(s)$, the curve $\gamma$ can be expressed by

$$
\gamma(s)=\int\left(\int \exp \left(\int k(s) d s\right) d s\right) d s \mathbf{b}+s \mathbf{c},
$$

where $\mathbf{c}$ is a unit space-like vector orthogonal to $\mathbf{b}$. In other words, $\gamma$ lies on a degenerate plane spanned by $\mathbf{b}$ and $\mathbf{c}$. We remark that $\langle T, \mathbf{b}\rangle=\langle N, \mathbf{b}\rangle=$ $\langle N, \mathbf{c}\rangle=0$ and $\langle T, \mathbf{c}\rangle=1$, i.e., $\gamma$ is a general helix and a slant helix.

Example 1. If $k=0$, then the principal normal vector field $N$ along $\gamma$ is constant and hence we have

$$
\gamma(s)=\frac{s^{2}}{2} N+s \mathbf{c}
$$

where $\mathbf{c}$ is a unit space-like vector orthogonal to $N$.

By putting $N=(1,1,0)$ and $\mathbf{c}=(0,0,1)$ and using the method of Duggal with $(-1,0, s)([10])$, we have the Frenet frame satisfying $(2.2)$ as follows:

$$
T(s)=(s, s, 1), N=(1,1,0) \text { and } B=\left(-\frac{1+s^{2}}{2}, \frac{1-s^{2}}{2}, s\right) \text {. }
$$

Note that $\langle T, N\rangle,\langle N, N\rangle,\langle N, \mathbf{c}\rangle$ and $\langle T, \mathbf{c}\rangle$ are constant, but $\langle B, N\rangle$ and $\langle B, \mathbf{c}\rangle$ are not constant. This arouses our curiosity whether the curve $\gamma$ is a binormal slant helix or not.

Now, for a space-like curve $\gamma$ of null type, we find a constant vector $V$ satisfying $\langle B, V\rangle$ is constant, say $a_{N}$. Consider a non-zero vector field $V$ along $\gamma$ as follows:

$$
V(s)=a_{T}(s) T(s)+a_{N} N(s)+a_{B}(s) B(s) .
$$

Differentiating (5.3), it follows that $V$ is a constant vector if and only if

$$
\left\{\begin{array}{l}
a_{T}^{\prime}(s)=a_{B}(s), \\
a_{T}(s)=-k(s) a_{N}, \\
a_{B}^{\prime}(s)=k(s) a_{B}(s) .
\end{array}\right.
$$

If $a_{N}=0$, then $a_{T}=a_{B}=0$, i.e., $V$ is zero vector, a contradiction. Therefore, $a_{N} \neq 0$. From (5.4), if $k=0$, then $V$ is zero vector which is also a contradiction. Thus, $k(s) \neq 0$.

By solving the ODE

$$
a_{N} k^{\prime}(s)+c \exp \left(\int k(s) d s\right)=0
$$



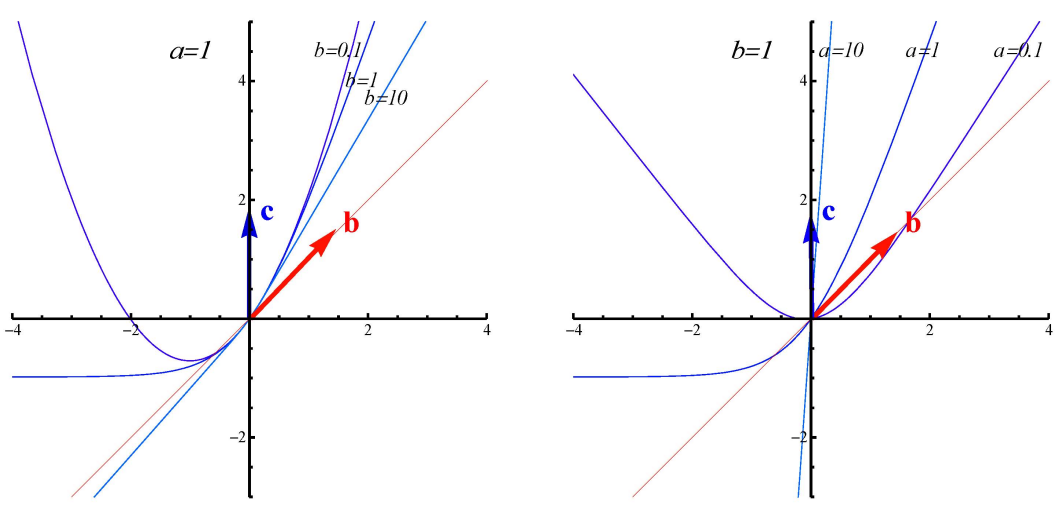

Fig 1. Degenerate plane curves of (5.9) with $a=1$ and $b=1$

for a non-zero constant $c$, we can find a constant vector $V$ along $\gamma$ satisfying

$$
a_{T}(s)=-k(s) a_{N} \text { and } a_{B}(s)=c \exp \left(\int k(s) d s\right) .
$$

Thus, $\gamma$ is a binormal slant helix if and only if (5.5) is satisfied.

Consequently, we have:

Theorem 5.2. A space-like curve $\gamma$ of null type in $\mathbb{E}_{1}^{3}$ is a binormal slant helix if and only if the curvature function $k$ of $\gamma$ is a solution of (5.5).

Corollary 5.3. A space-like curve $\gamma$ of null type in $\mathbb{E}_{1}^{3}$ with constant curvature $k$ is not binormal slant helix.

Example 2. Assume that $k^{\prime}(s) \neq 0$. Put $K(s)=\exp \left(\int k(s) d s\right)$. If $c / a_{N}>0$, then we have a solution $K(s)$ of $(5.5)$ given by

$$
K(s)=\operatorname{sech}^{2}(b s),
$$

where $b=\sqrt{\left|\frac{c}{2 a_{N}}\right|}$, from which, we have a solution $k(s)$ of $(5.5)$ given by

$$
k(s)=-2 b \tanh (b s) .
$$

By putting $\mathbf{b}=(1,1,0)$ and $\mathbf{c}=(a, a, 1)$, from $(5.1)$ we have a binormal slant helix $\gamma$ as follows:

$$
\gamma(s)=\left(\frac{1}{b^{2}} \ln \cosh (b s)+a s, \frac{1}{b^{2}} \ln \cosh (b s)+a s, s\right) .
$$

For this curve $\gamma$, from (5.6) we can give a constant vector $V$ as follows:

$$
V=\frac{1}{2 b^{2}}\left(1-b^{2}-a^{2} b^{2}, 1+b^{2}-a^{2} b^{2},-2 a b^{2}\right) \text {. }
$$


Corollary 5.4. A null curve in $\mathbb{E}_{1}^{3}$ parametrized by the pseudo-arc parameter is the same as its null binormal-directional curve up to Lorenzian translation if and only if its light-like curvature equals 1 or -1 .

\section{References}

[1] A. T. Ali, Position vectors of spacelike general helices in Minkowski 3-space, Nonlinear Anal. 73 (2010), no. 4, 1118-1126.

[2] A. T. Ali and R. Lopez, Timelike $\mathrm{B}_{2}$-slant helices in Minkowski $\mathbb{E}_{1}^{4}$, Arch. Math. (Brno) 46 (2010), no. 1, 39-46.

[3] $159-167$.

[4] A. T. Ali and M. Turgut, Position vector of a time-like slant helix in Minkowski 3-space, J. Math. Anal. Appl. 365 (2010), no. 2, 559-569.

[5] _ Determination of time-like general helices in Minkowski 3-space, Preprint 2009: arXiv:0906.3851v1 [math.DG].

[6] A. Bejancu, Lightlike curves in Lorentz manifolds, Publ. Math. Debrecen 44 (1994), no. $1-2,145-155$.

[7] J. H. Choi and Y. H. Kim, Associated curves of a Frenet curve and their applications, Appl. Math. Comput. 218 (2012), 9116-9124.

[8] J. H. Choi, Y. H. Kim, and A. T. Ali, Some associated curves of a Frenet non-lightlike curve in $\mathbb{E}_{1}^{3}$, J. Math. Anal. Appl. 394 (2012), 1285-1296.

[9] K. L. Duggal and A. Bejancu, Lightlike Submanifolds of Semi-Riemannian Manifolds and Applications, Kluwer, Dordrecht, The Netherlands, 1996.

[10] K. L. Duggal and D. H. Jin, Null Curves and Hypersurfaces of Semi-Riemannian Manifolds, World Scientific, 2007.

[11] A. Ferrandez, A. Gimenez, and P. Lucas, Null helices in Lorentzian space forms, Internat. J. Modern Phys. A 16 (2001), no. 30, 4845-4863.

[12] _ Null generalized helices in Lorentzian-Minkowski spaces, J. Phys. A: Math. Gen. 35 (2002), 8243-8251.

[13] L. P. Hughston and W. T. Shaw, Real classical string, Proc. Roy. Soc. London Ser. A 414 (1987), no. 1847, 415-422.

[14] _ Classical strings in ten dimensions, Proc. Roy. Soc. London Ser. A 414 (1987), no. $1847,423-431$.

[15] — Constraint-free analysis of relativistic strings, Classical Quantum Gravity 5 (1988), no. 3, 69-72.

[16] _ Constraint-free analysis of relativistic strings, Class. Quant. Grav. 5 (1988), 69-72.

[17] _ Twistors and strings, Amer. Math. Soc. RI 1988, 337-363.

[18] Spinor parametrizations of minimal surfaces, The mathematics of surfaces, III (Oxford, 1989), 359-372, Inst. Math. Appl. Conf. Ser. New Ser., 23, Oxford Univ. Press, New York, 1989.

[19] J. I. Inoguchi and S. W. Lee, Null curves in Minkowski 3-space, Int. Electron. J. Geom. 1 (2008), no. 2, 40-83.

[20] H. B. Karadağ and M. Karadağ, Null generalized slant helices in Lerontzian space, J. Phys. A: Math. Gen. 35 (2002), 8243-8251.

[21] L. Kula and Y. Yayli, On slant helix and its spherical indicatrix, Appl. Math. Comp. 169 (2005), no. 1, 600-607.

[22] B. O'Neill, Semi-Riemannian Geometry with Application to Relativity, Academic Press, New York, 1983. 
JiN Ho ChOI

Department of Mathematics

UNIVERSITY OF ULSAN

ULSAN 680-749, Korea

E-mail address: jino7142@knu.ac.kr

Young Ho Kim

Department of Mathematics

Kyungpook National University

TAEGU 702-701, Korea

E-mail address: yhkim@knu.ac.kr 